\title{
A forgotten group during humanitarian crises: a systematic review of sexual and reproductive health interventions for young people including adolescents in humanitarian settings
}

\author{
Lauren Jennings ${ }^{1}$, Asha S. George ${ }^{2}$, Tanya Jacobs ${ }^{2}$, Karl Blanchet ${ }^{1}$ and Neha S. Singh ${ }^{1 *}$ (i)
}

\begin{abstract}
Background: Young people including adolescents face barriers to healthcare and increased risk of poor sexual and reproductive health (SRH), which are exacerbated in humanitarian settings. Our systematic review assessed the evidence on SRH interventions for young people including adolescents in humanitarian settings, strategies to increase their utilisation and their effects on health outcomes.

Methods: We searched peer-reviewed and grey literature published between 1980 and 2018 using search terms for adolescents, young people, humanitarian crises in low- and middle- income countries and SRH in four databases and relevant websites. We analysed literature matching pre-defined inclusion criteria using narrative synthesis methodology, and appraised for study quality.

Findings: We found nine peer-reviewed and five grey literature articles, the majority published post-2012 and mostly high- or medium-quality, focusing on prevention of unintended pregnancies, HIV/STIS, maternal and newborn health, and prevention of sexual and gender-based violence. We found no studies on prevention of mother-to-child transmission (PMTCT), safe abortion, post-abortion care, urogenital fistulae or female genital mutilation (FGM). Thirteen studies reported positive effects on outcomes (majority were positive changes in knowledge and attitudes), seven studies reported no effects in some SRH outcomes measured, and one study reported a decrease in number of new and repeat FP clients. Strategies to increase intervention utilisation by young people include adolescent-friendly spaces, peer workers, school-based activities, and involving young people.
\end{abstract}

\footnotetext{
* Correspondence: neha.singh@lshtm.ac.uk

${ }^{1}$ Health in Humanitarian Crises Centre, London School of Hygiene and

Tropical Medicine, 15-17 Tavistock Place, London WC1H 9SH, UK

Full list of author information is available at the end of the article
}

(c) The Author(s). 2019 Open Access This article is distributed under the terms of the Creative Commons Attribution 4.0 International License (http://creativecommons.org/licenses/by/4.0/), which permits unrestricted use, distribution, and reproduction in any medium, provided you give appropriate credit to the original author(s) and the source, provide a link to the Creative Commons license, and indicate if changes were made. The Creative Commons Public Domain Dedication waiver (http://creativecommons.org/publicdomain/zero/1.0/) applies to the data made available in this article, unless otherwise stated. 
(Continued from previous page)

Discussion: Young people, including adolescents, continue to be a neglected group in humanitarian settings. While we found evidence that some SRH interventions for young people are being implemented, there are insufficient details of specific intervention components and outcome measurements to adequately map these interventions. Efforts to address this key population's SRH needs and evaluate effective implementation modalities require urgent attention. Specifically, greater quantity and quality of evidence on programmatic implementation of these interventions are needed, especially for comprehensive abortion care, PMTCT, urogenital fistulae, FGM, and for LGBTQI populations and persons with disabilities. If embedded within a broader SRH programme, implementers and/or researchers should include young people-specific strategies, targeted at both girls/women and boys/men where appropriate, and collect age- and sex-disaggregated data to help ascertain if this population's diverse needs are being addressed.

Keywords: Adolescent health, Young people, Sexual health, Reproductive health, Humanitarian, Conflict, Crises, Emergencies, Systematic review

\section{Introduction}

In 2018, the United Nations (UN) estimated that 135.7 million people were in need of humanitarian aid in 25 countries around the world [1]. The United Nations High Commissioner for Refugees (UNHCR) reports that this large population includes 70.8 million people who have been forcibly displaced from their homes because of conflict and persecution, including 25.9 million refugees, over half of whom are under the age of 18 [2]. These populations are more likely to have poor health outcomes, including in sexual and reproductive health (SRH), due to disrupted services, lack of health supplies, scarcity of trained health workers, and increased risk of sexual violence [3]. Young people in these settings will often find themselves in high-risk situations and may be forced to take on adult roles within their families and communities [4].

Adolescents are defined by the World Health Organization (WHO) as persons aged 10-19 years. However, research concerning adolescents is often extended to include persons aged 10-24 years, defined as 'young people' [5]. While young people are not a homogenous group, they are a vulnerable population group and over the past two decades, increased attention has been paid to addressing their unique health needs [6]. This population undergo an intense period of physical, cognitive, emotional and social development, setting them apart from children and adults [7]. This rapid development results in new behaviours, which can have an impact on short- and long-term health outcomes [8], requiring a tailored health care approach [7].

Despite the specific needs and vulnerabilities faced by young people, most services are not organised to recognise or meet these needs [7]. For example, young people often face barriers when accessing health care including a lack of knowledge about their health and health services, an inability to travel to access these services, restrictive laws and judgemental attitudes of health care workers [7, 9, 10]. Additionally, young people are in a period of their life with increased need for privacy and confidentiality with a greater fear of embarrassment and judgement by others [9]. They therefore require services that are respectful and responsive to their needs [9].

The vulnerability of young people in humanitarian settings is compounded. As families, communities and social groups are disrupted in these settings [5], adolescents may find themselves in high-risk situations and may be forced to take on adult roles within their families and communities [3]. There can also be interruption of adolescent SRH service delivery resulting in a lack of access to and information about available services and an increase in the risk of sexual exploitation and abuse [11]. It is for this reason that particular emphasis needs to be placed on making $\mathrm{SRH}$ services in humanitarian settings young peopleinclusive and tailored towards their specific needs. At the International Conference on Population and Development (ICPD) in Cairo in 1994, the SRH rights of those living in crisis-affected settings were first recognised [12]. This led to the formation of the Inter-Agency Working Group (IAWG) for Reproductive Health in Refugee Settings and the development of the Inter-Agency Field Manual (IAFM) for Reproductive Health in Humanitarian Settings [13-15]. The IAFM introduces the Minimum Initial Services Package (MISP), which is a set of priority interventions (Panel 1) and actions designed to reduce morbidity and mortality and provide guidelines for coordinated $\mathrm{SRH}$ services in the early stages of an emergency as well as guidelines for comprehensive services once the situation has stabilised [13, 14].

The IAFM also includes a chapter on adolescent SRH which, along with the Adolescent Sexual and Reproductive Health Toolkit for Humanitarian Settings published by the United Nations Population Fund (UNFPA) [5], provides guidance on making SRH services in these settings inclusive to adolescents and young people.

Despite this guidance, there has been no systematic review focusing solely on SRH interventions for young 
people in humanitarian settings. A previous review on SRH interventions in humanitarian settings in the general population found four interventions that targeted adolescents, all of which were HIV prevention interventions [16]. Similarly, Warren et al (2015) found generally low-quality evidence on the effectiveness of SRH interventions in humanitarian settings and did not find any young people-focused studies [3]. Singh et al (2018) assessed the utilisation of SRH services in humanitarian crises and found only one study targeted to this population [17].

Young people are a key population in humanitarian settings, and yet little is known about the evaluation of SRH interventions directed to them. In order to address these gaps in knowledge, our systematic review aims to assess the evidence on the spectrum of SRH interventions being delivered to young people including adolescents in humanitarian settings, as well as the strategies to increase their utilisation and their effects on health outcomes.

\section{Methods}

This systematic review follows the reporting guidelines as set out in the Preferred Reporting Items for Systematic Reviews and Meta-Analyses (PRISMA) statement [18]. Table 1 describes the inclusion and exclusion criteria.

We defined a humanitarian setting as one in which 'an event or series of events has resulted in a critical threat to health, safety, security or well-being of a community or other large group of people'. [15] The affected community is no longer able to cope and external assistance, whether from the national or international level, is required. The event can be a natural or man-made disaster [15], and settings can range from acute to stabilised. While we recognise that some forcibly-displaced populations live in stable, high-income settings, we focused only on interventions implemented in low- and middleincome countries (LMIC), as the majority of humanitarian settings occur in LMIC and the resources available in highincome countries to deal with humanitarian emergencies are different and much greater than those in LMIC [3].

Both peer-reviewed and grey literature were included. All quantitative and qualitative research studies describing an SRH intervention for young people including adolescents and measuring an SRH outcome were included. Outcomes of interest were based on those outlined in the MISP as part of the IAWG Field Manual, focusing on measures of prevention of sexual violence, prevention of transmission and reduction of morbidity and mortality related to HIV and other STIs, prevention of excess maternal and newborn morbidity and mortality, prevention of unintended pregnancies, and safe abortion [13].

\section{Search strategy}

Our search strategy focused on literature published between 1980 and 2018. It was adapted from previous systematic reviews on SRH in humanitarian settings $[3,17]$ and finalised with help from librarians trained in systematic review methodology.

The search included terms in the following categories: 1) humanitarian settings and crises, 2) LMIC, 3) SRH interventions, and 4) young people including adolescents. Both free-text searching and subject headings were used. The full search strategy can be found in the Additional file 1 .

The search included peer-reviewed literature retrieved from the following databases: MEDLINE, EMBASE, Global Health and PsycINFO. A grey literature search was conducted using the following online resources and websites: Results for Development, Reproductive Health Response in Crisis Consortium, Médecins Sans Frontières, UNFPA, RAISE initiative, IAWG, Save the Children, The International Rescue Committee (IRC), CARE International, International Committee of the Red Cross, International Planned Parenthood Federation, AIDS Alliance, Marie Stopes International, Women's Refugee Commission (WRC), Population Council and The Coalition for

Table 1 Inclusion and exclusion criteria for the systematic review

\begin{tabular}{|c|c|c|}
\hline Category & Included & Excluded \\
\hline Population of interest & $\begin{array}{l}\text { Young people including adolescents (male and female) } \\
\text { aged 10-24 years living in humanitarian settings in } \\
\text { low- and middle-income countries (LMICs) }\end{array}$ & $\begin{array}{l}\text { Populations living in humanitarian settings } \\
\text { in high-income countries }\end{array}$ \\
\hline Intervention & $\begin{array}{l}\text { Any intervention aimed at improving SRH outcomes as } \\
\text { defined in the Minimum Initial Service Package as part } \\
\text { of the Inter-Agency Field Manual for Reproductive Health } \\
\text { in Humanitarian Settings [13] }\end{array}$ & \\
\hline Article type & $\begin{array}{l}\text { Any quantitative or qualitative study describing an SRH } \\
\text { intervention and measuring an SRH outcome. }\end{array}$ & $\begin{array}{l}\text { Any study with no specific SRH intervention } \\
\text { or that only describes needs, prevalence or } \\
\text { risk factors. }\end{array}$ \\
\hline Crisis type & $\begin{array}{l}\text { Any acute or protracted armed conflict, disease outbreak, } \\
\text { or natural disaster }\end{array}$ & Studies conducted before a crisis has occurred \\
\hline Publication date & 1980-2018 & \\
\hline Language & English, French & Other languages \\
\hline
\end{tabular}


Adolescent Girls. Broad search terms such as 'young', 'adolescent', 'humanitarian' and 'sexual health' as well as topic-based searches were used. Advanced searches using similar terms were run on Google Scholar and the Popline database.

Reference lists of included peer-reviewed and grey literature publications and relevant systematic reviews were screened for additional articles. Experts in the field of adolescent SRH in humanitarian settings, including members of IAWG's adolescent SRH sub-working group, were contacted to identify literature not found during the systematic search. Only literature from 1980 to 2018 was searched as a previous review found no SRH studies published prior to that date [19].

\section{Study selection and data extraction}

All citations from the database searches were exported to Mendeley referencing software and then to Excel. LJ and NSS conducted the screening independently (Fig. 1) using pre-defined inclusion and exclusion criteria (Table 1). Data from the included interventions were extracted under the following headings: author and year, study setting, target population, crisis type, SRH domain, study design, study outcomes, intervention description, results, implementing bodies and funders.

\section{Data analysis}

Due to the heterogeneity of interventions, settings, and outcomes measured, we used a narrative synthesis approach to analyse findings [20]. Findings were reviewed and synthesised by the lead author (LJ) following a process of constant comparison. After drafting synthesised findings, authors (LJ, NSS) revisited the original articles to check their interpretations.

The quality of included articles was assessed using critical appraisal checklists appropriate to the type of publication, described in Table 2.

All articles were given a final quality score which was converted into a percentage of the total achievable score. Articles were then given a rating of low-, medium- or high-quality based on that percentage. Low-quality studies scored between 0 and 33\%, medium-quality scored between 34 and 66\% and high-quality studies scored $67 \%$ and above. These quality thresholds have been used

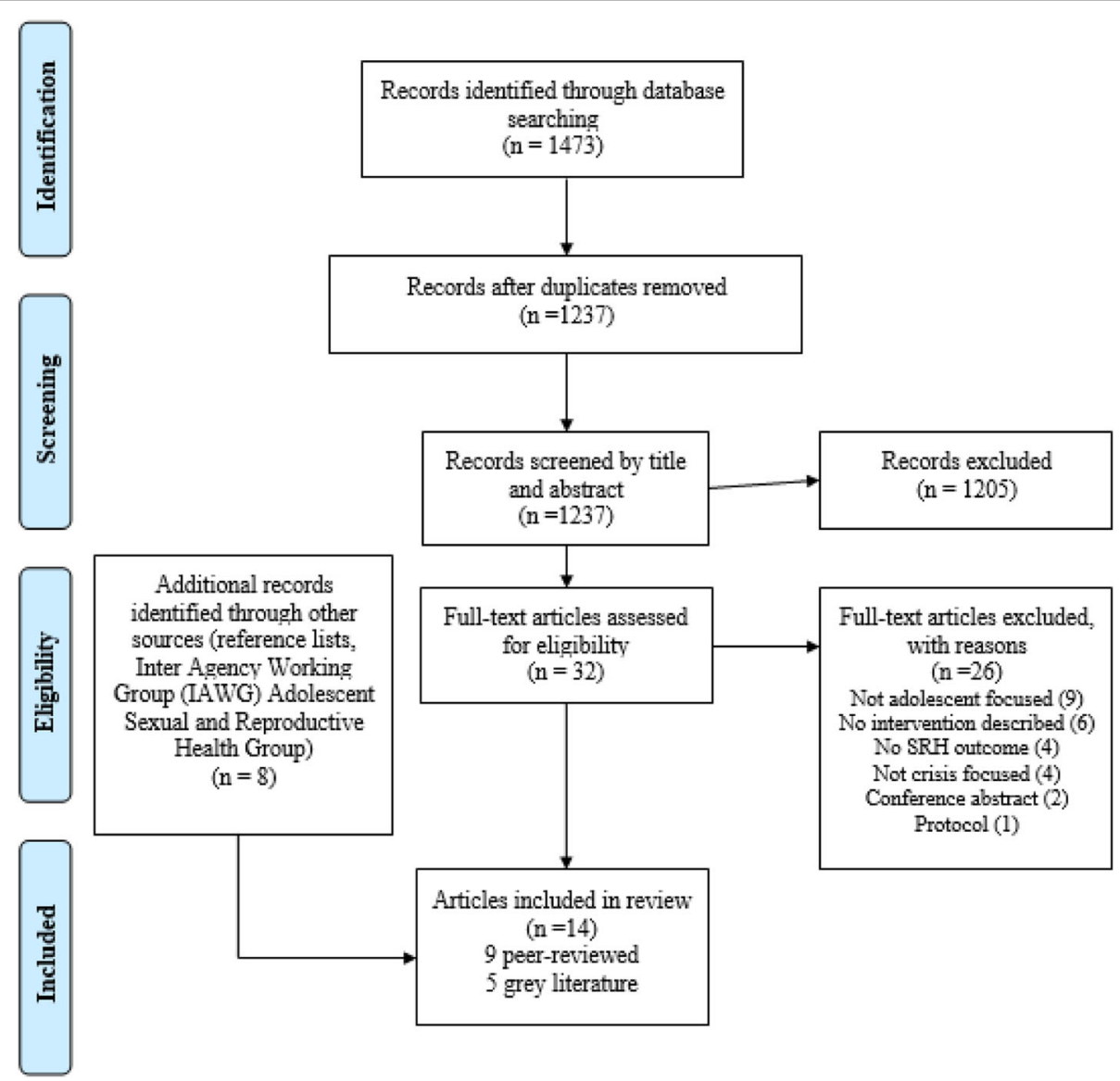

Fig. 1 PRISMA flow-chart [17] for systematic review on sexual and reproductive health interventions for young people including adolescents in humanitarian crises settings 
Table 2 Quality appraisal checklists used in the systematic review

\begin{tabular}{ll}
\hline Study design & Quality appraisal checklist \\
\hline Randomised control & $\begin{array}{l}\text { Consolidated Standard of Reporting } \\
\text { trials (RCTs) }\end{array}$ \\
Trials (CONSORT) checklist [21] & $\begin{array}{l}\text { Strengthening of Observational studies } \\
\text { in Epidemiology (STROBE) checklist [22] }\end{array}$ \\
Before- and after-study & $\begin{array}{l}\text { National Heart, Lung and Blood Institute's } \\
\text { checklist [23] }\end{array}$ \\
Qualitative study & $\begin{array}{l}\text { Critical Appraisal Skills Programme (CASP) } \\
\text { checklist [24] }\end{array}$ \\
Case study & $\begin{array}{l}\text { Centre for Evidence-Based Medicine } \\
\text { checklist [25] }\end{array}$ \\
Grey literature & $\begin{array}{l}\text { Authority, accuracy, coverage, objectivity, } \\
\text { date, significance (AACODS) checklist [26] }\end{array}$ \\
\end{tabular}

in previous reviews of SRH interventions in humanitarian settings [3, 17].

\section{Results}

\section{Overview of studies}

We screened titles and abstracts of 1237 records from peer-reviewed databases and 8 articles from other sources. After full-text screening, 14 articles (i.e. 9 peerreviewed studies [27-35] and 5 grey literature articles [36-40]) met our inclusion criteria and were included in the review (Fig. 1). Two grey literature articles described more than one SRH intervention for young people, and several interventions were described in more than one article, so we present findings from 15 individual SRH interventions for young people in humanitarian settings (Table 3). Of the 14 articles included in the review, 13 were published between 2012 and 2018, and one was published before 2012, in 2006 [27].

\section{Study design and quality}

The nine peer-reviewed articles included three randomised controlled trials (RCTs) [28, 32, 33], one cohort study [30], two case studies [34, 35], one before-andafter study [27], and two qualitative studies [29, 31] (Table 3$)$. Of the grey literature articles $(n=5)$, four were case studies [37-40] and one presented two RCTs and a before-and-after study [39].

Among the peer-reviewed literature, all three RCTs were considered to be medium-quality [28, 32, 33] (Table 3), as none of them adequately described sample size calculation or the randomisation process. The observational study [30] included was high-quality despite inadequate consideration of confounders in the analysis. Of the two qualitative studies, one was high-quality and the other of low-quality. The three remaining peerreviewed articles were found to be medium-quality $[27,34,35]$. Among the grey literature articles, three were found to be high-quality [37-39], while two were considered medium-quality [36, 40].

\section{Study setting}

One intervention was delivered in an acute disease outbreak setting, and three in natural disaster settings (Table 3). The remaining 11 interventions were delivered in areas affected by armed conflict, either in protracted violence or post-conflict settings. Nine interventions were implemented in Sub-Saharan Africa (SSA), with one of these interventions also being implemented in Pakistan. Three interventions were implemented only in Asia, while the remaining interventions were implemented in South America $(n=2)$ and the Middle East $(n=1)$.

\section{Target population}

All interventions targeted adolescents, with five extending the eligible age group to include young people [27, 30, 38] (Table 3). Only four interventions were explicitly inclusive of very young adolescents aged 10-14 years [37-39]. Seven interventions did not specify an age-range but mentioned targeting adolescents. Nine interventions targeted both males and females though only one study provided differentiated results [27]. While all interventions were inclusive of girls or young women, there were no interventions targeted exclusively at adolescent boys or young men.

\section{Overview of SRH interventions \\ SRH domains covered by the interventions}

Over half $(n=9)$ of the 15 interventions included in the review provided more than one element of $\mathrm{SRH}$ as part of their interventions to reach adolescents and young people in humanitarian settings (Table 3 ). The included studies focused on prevention of unintended pregnancies $(n=8)$, prevention of the transmission of and morbidity and mortality related to HIV and STIs $(n=8)$, prevention of excess maternal and newborn morbidity and mortality $(n=4)$, and prevention of sexual and genderbased violence (SGBV) $(n=2)$. We identified no studies focused on prevention of mother-to-child transmission (PMTCT), safe abortion, post-abortion care, urogenital fistulae or female genital mutilation (FGM). Furthermore, we found no studies targeting LGTBQI populations or young people with disabilities.

\section{Strategies to increase utilisation of SRH services Adolescent-friendly spaces}

Eight interventions described having adolescent- or youth-friendly services as part of their intervention [29, 36-38] (Table 3). However, adolescent-friendly services were not always defined and it is therefore difficult to determine if this was actually achieved. Four case studies utilised an adapted version of the Inter-Agency Field Manual Checklist (Table 4) to assess if an intervention was adolescent-friendly, and were found to fulfil the majority of the criteria $[37,38]$. 


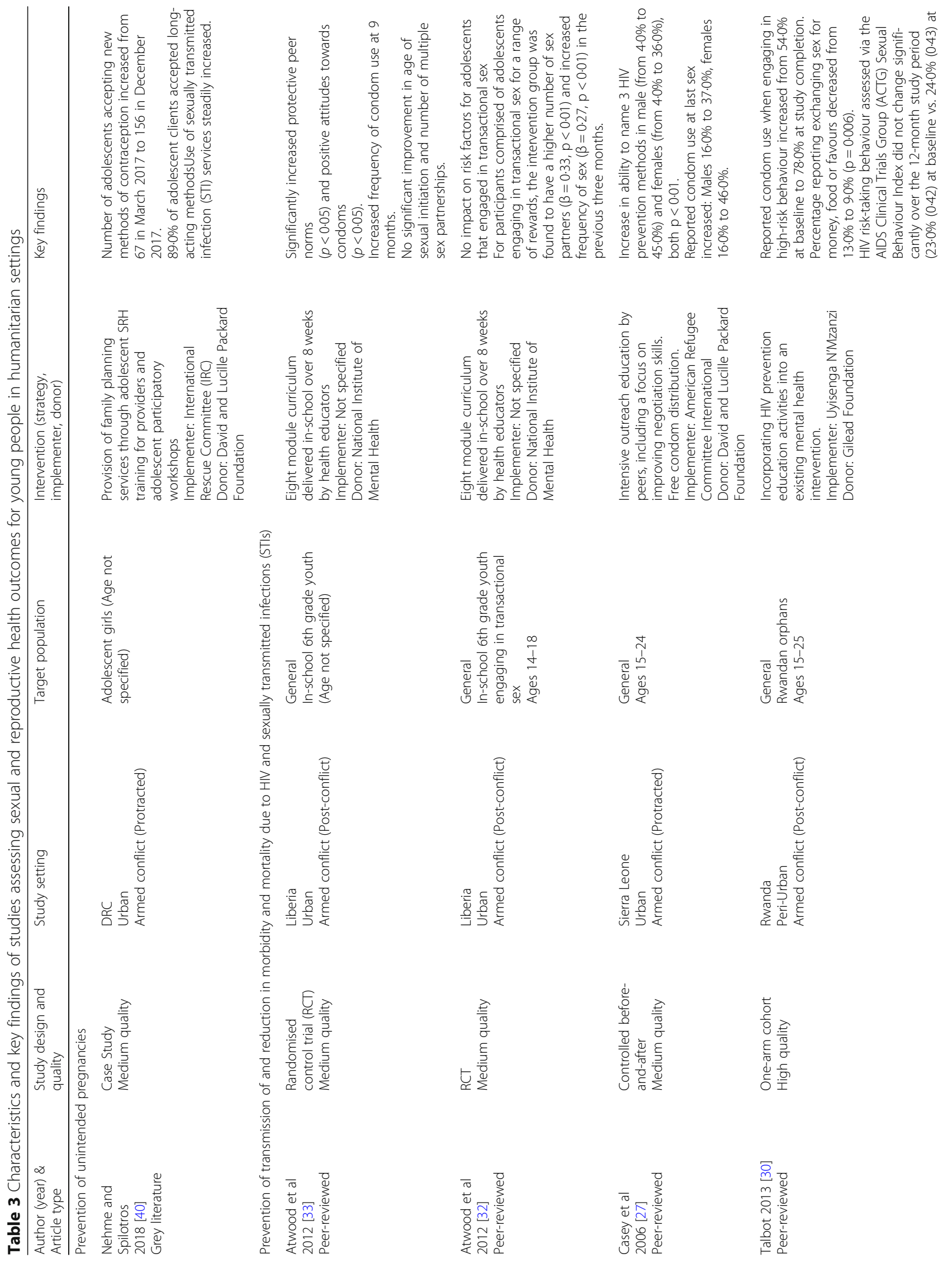




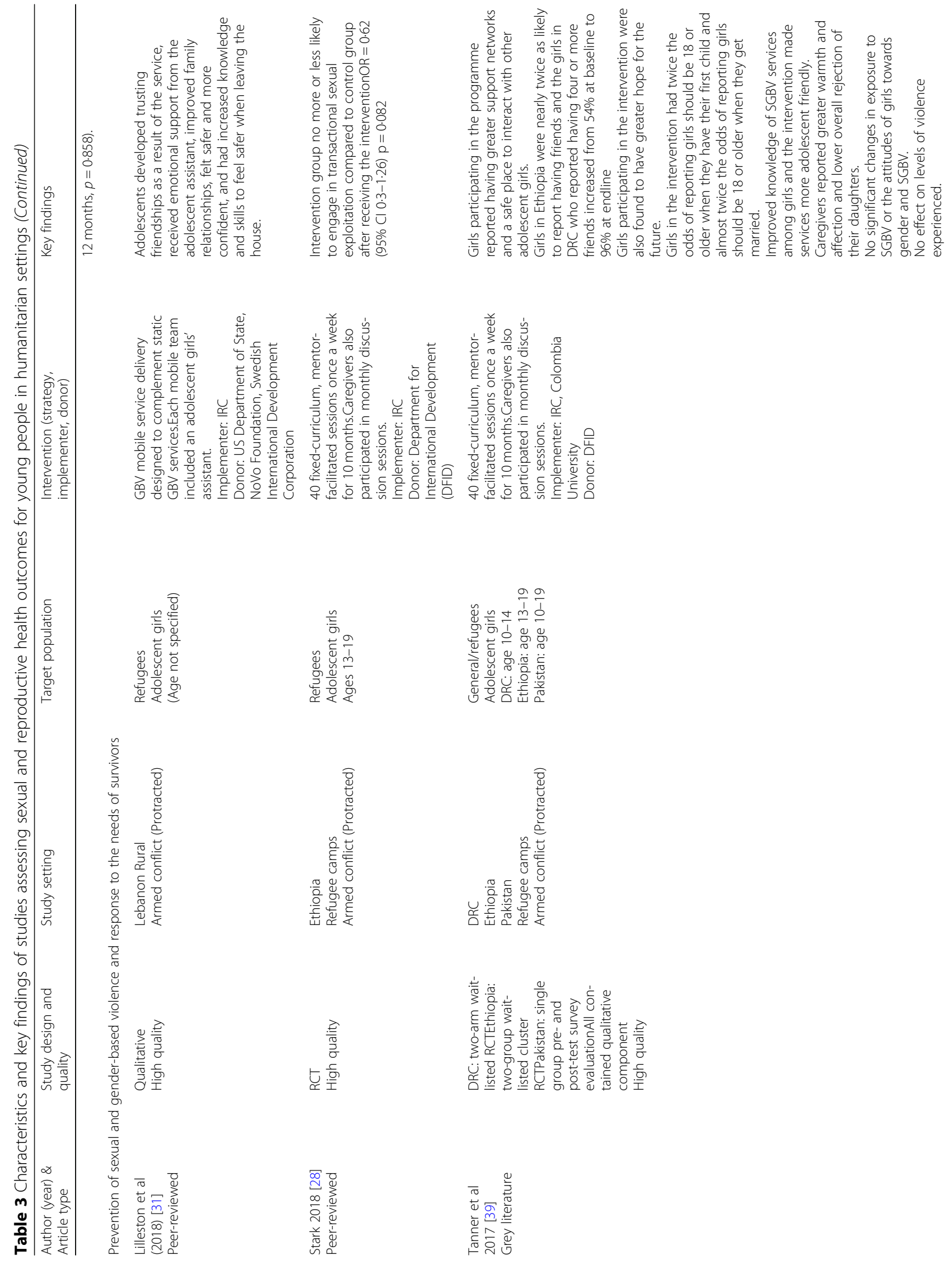




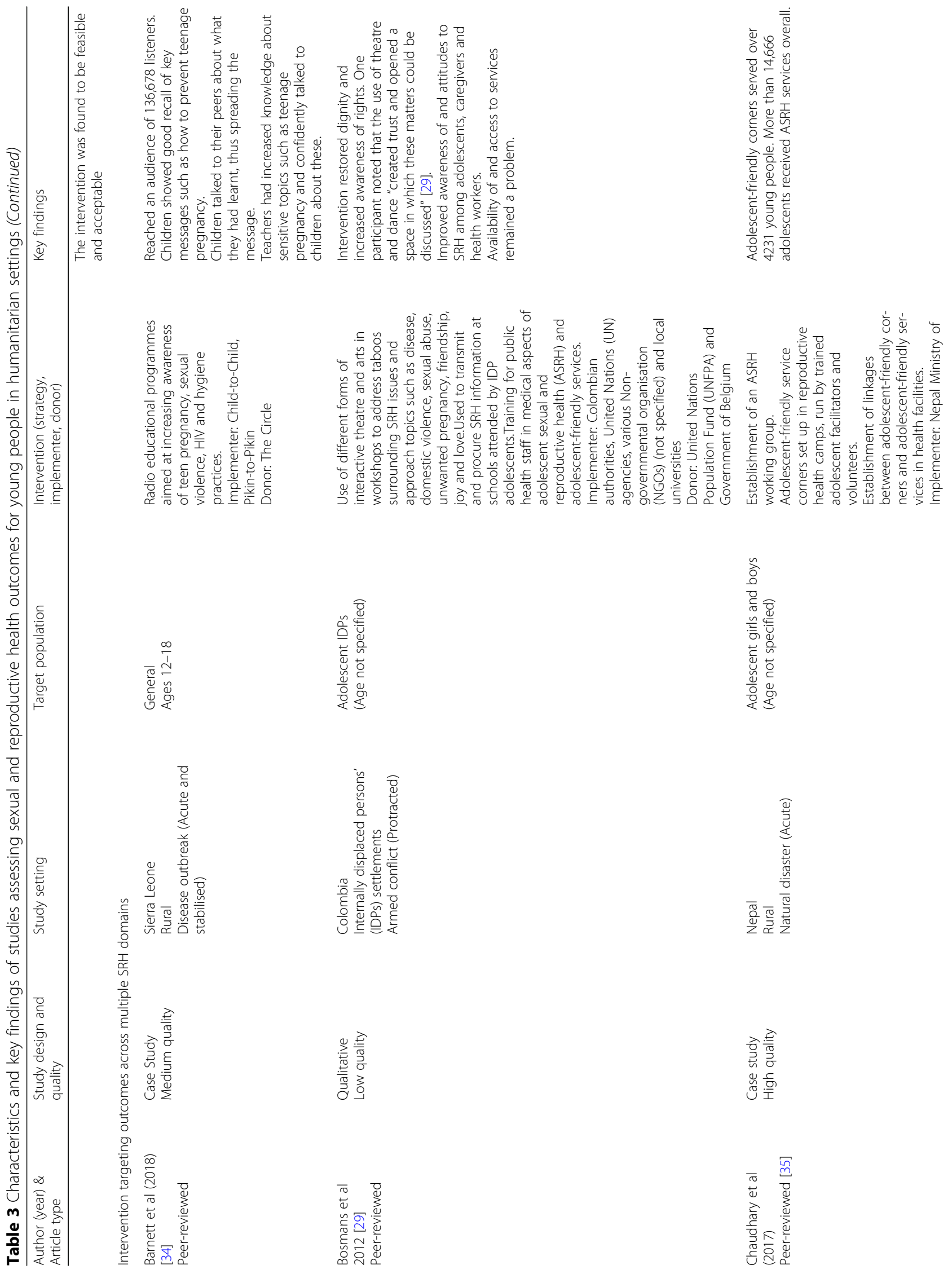




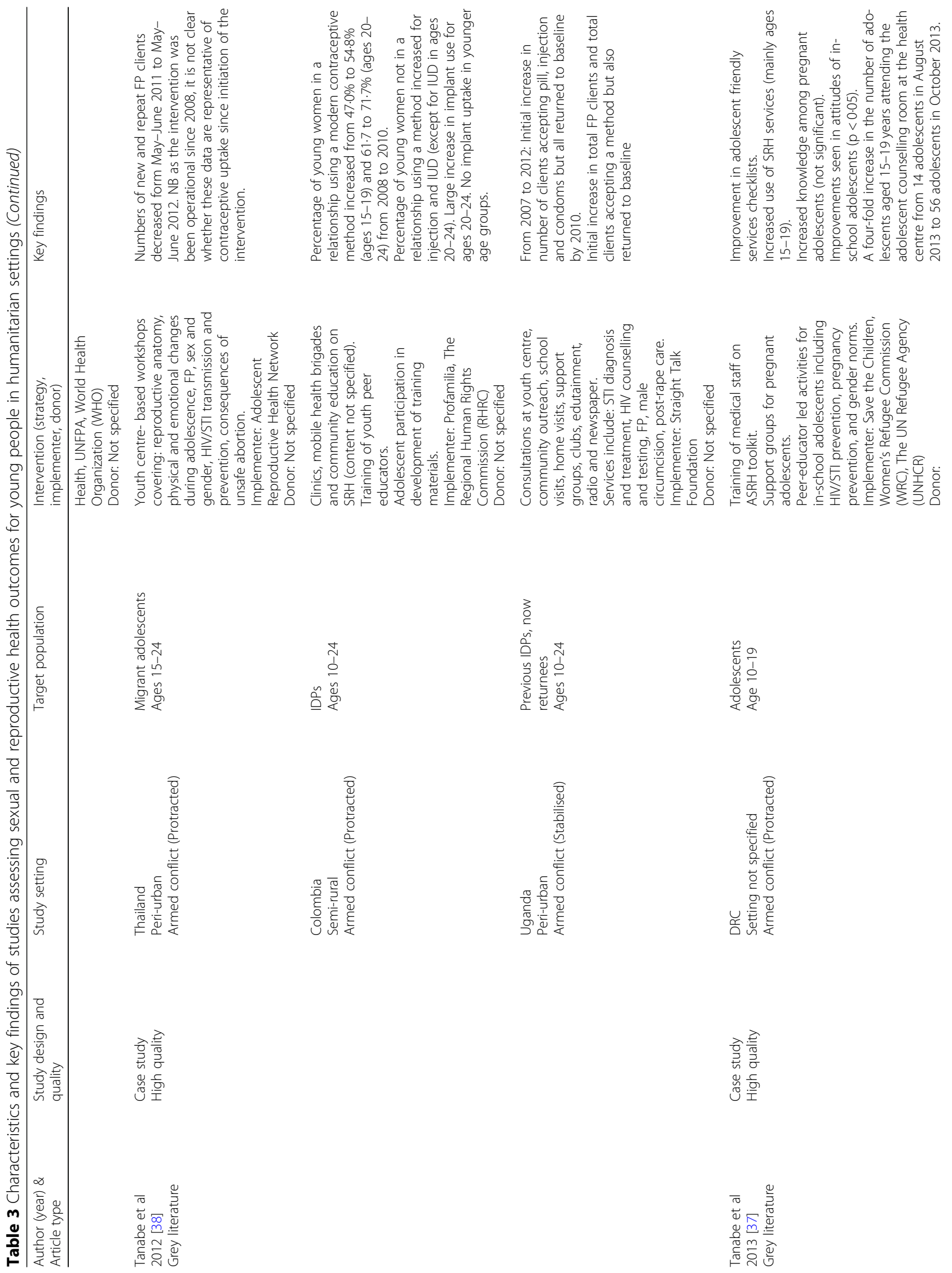




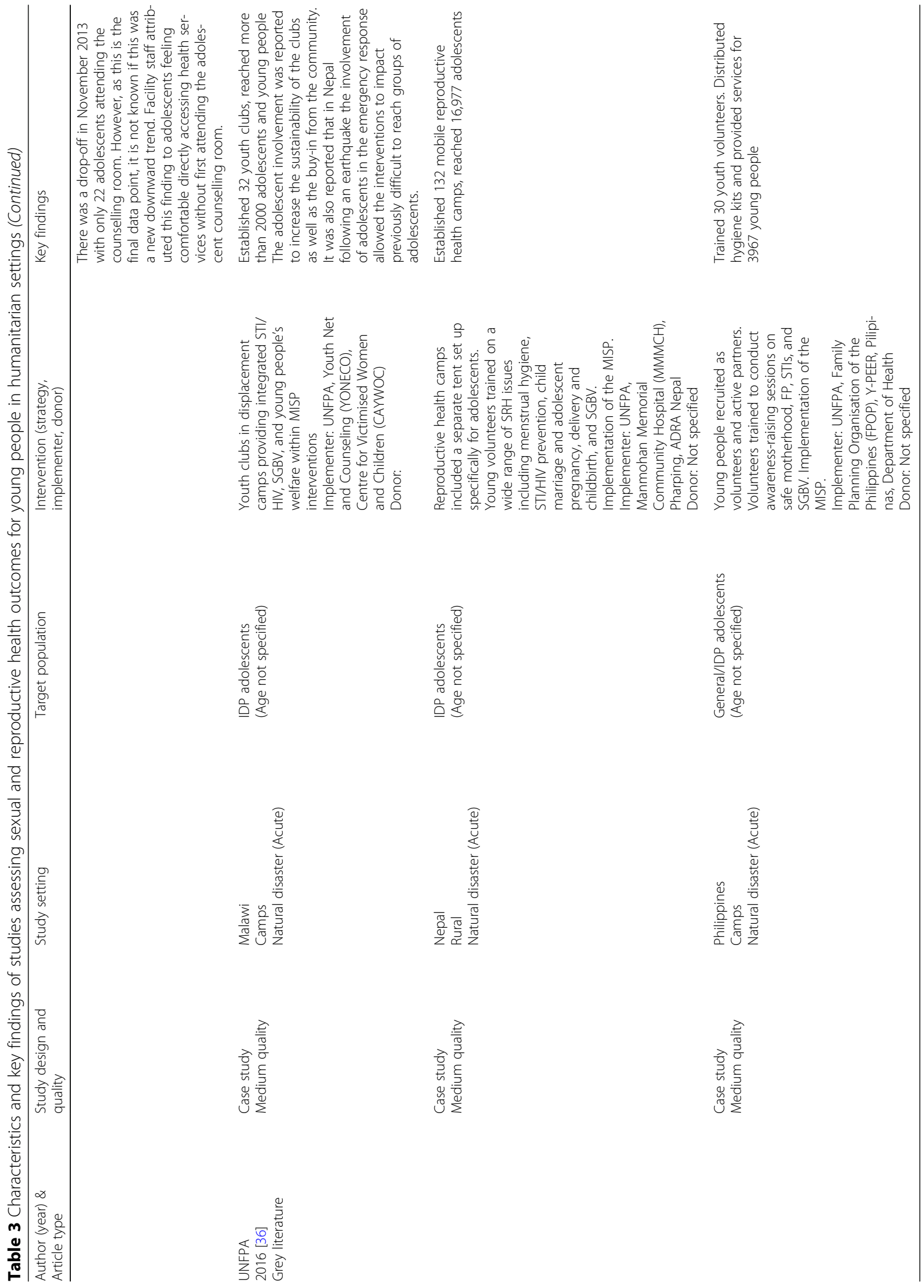


Table 4 Adapted Version of the Inter-Agency Field Manual Checklist for Adolescent Friendly Services [37, 38]

\begin{tabular}{|c|c|}
\hline \multicolumn{2}{|c|}{ Adolescent-Friendly Checklist } \\
\hline Health Facility & $\begin{array}{l}\text { - Convenient hours } \\
\text { - Convenient location } \\
\text { - Adequate space and sufficient privacy } \\
\text { - Comfortable surroundings }\end{array}$ \\
\hline Provider & $\begin{array}{l}\text { - Respect for adolescents } \\
\text { - Non-judgmental attitude } \\
\text { - Privacy and confidentiality honoured } \\
\text { - Peer counselling available } \\
\text { - Same-sex providers when possible } \\
\text { - Strict confidentiality maintained } \\
\text { - Staff trained in youth-friendly health service } \\
\text { characteristics }\end{array}$ \\
\hline Administrative & $\begin{array}{l}\text { - Adolescent involvement } \\
\text { - Boys and young men welcome } \\
\text { - Necessary referrals available } \\
\text { - Affordable fees } \\
\text { - Drop-in clients welcome } \\
\text { - Publicity and recruitment that informs and reassures } \\
\text { adolescents }\end{array}$ \\
\hline
\end{tabular}

\section{Peer workers}

Casey et al (2006) described an intervention in a protracted armed conflict setting in Sierra Leone targeting male and female young people and provided intensive peer outreach education in order to improve condom negotiating skills, and free distribution of male condoms by peer workers [27].

Tanabe et al (2013) described using peer educators to conduct outreach and sensitisation to SRH issues including but not limited to prevention of unintended pregnancies, and prevention of HIV and STIS, in the community [38]. In Colombia, Tanabe et al (2012) used peer workers to increase community support for SRH interventions for young people and target vulnerable populations who might otherwise have difficulty accessing services [38]. In Thailand, Tanabe et al. (2012) also used peer educators to educate migrant youth on SRH issues [38].

The Creating Opportunities through Mentoring, Parental Involvement and Safe Spaces (COMPASS) intervention implemented in the DRC, Ethiopia and Pakistan focused on prevention of SGBV by using older girls aged 18-30 to act as mentors and facilitate a fixed-curriculum programme targeted to adolescent girls and their parents or caregivers, with an aim of reducing SGBV in girls aged 10-19 [28, 39]. The mentors came from a similar background to the participants and in Pakistan, they were former participants of the programme.

Using peers as part of an intervention did present some challenges. All three case studies by Tanabe et al (2012) reported that retention of peer workers is a challenge, especially as they often work as volunteers and lack incentive to continue, which can affect the sustainability of the intervention [38]. A concern arising from the COMPASS intervention was that, as peer workers were from the same background as adolescents participating in the programme, they might reinforce harmful societal norms regarding gender roles and gender-based violence [39].

\section{Involving adolescents}

IRC piloted an intervention in the DRC designed to increase uptake of contraception among adolescent girls through a participatory approach [40]. Adolescent girls were included at all stages of implementing the intervention, from workshops aimed at identifying and setting priorities for adolescent SRH services, to forming coordination committees for the implementation of the decided-upon actions. They also participated in progress monitoring and supervision visits to facilities. However, they did not participate in the overall research process.

In response to floods in Malawi, adolescents were involved in planning and implementing youth clubs within displacement camps. These youth clubs aimed to provide a range of SRH services in an integrated approach to adolescent girls and boys [36].A youth centre in Uganda also used adolescent input in its design and employed community outreach and home visits to deliver SRH care [38].

\section{Engaging communities}

Tanabe et al (2012)'s case studies from Thailand, Uganda and Colombia reported initial resistance from adults in the community to the provision of SRH services to adolescents, mostly expressed through a fear that SRH activities might encourage young people to engage in sexual activity [38]. The three interventions reported successfully overcoming this resistance by building trust with the community through engagement of community leaders [38].

\section{School-based activities}

The Making Proud Choices intervention in post-conflict Liberia targeted in-school 6th grade youth and provided an eight module training programme delivered in school by health educators, which aimed to prevent HIV by promoting positive attitudes towards condom use and increasing skills required to negotiate safer sex practices [32, 33].

A case study from the DRC targeted in-school adolescents aged 12-14 years [37]. Most of the activities occurred within the schools and involved the teachers being trained to "champion" adolescent SRH [37]. Peer educators were selected by their classmates and trained on various SRH topics including HIV and STI prevention, menstruation, and puberty. Peer education sessions were mainly conducted outside of normal school hours, however, many in-school adolescents were unable to attend as 
they were expected to be home immediately after school, demonstrating the need for school-based activities to be conducted within regular school hours [37].

\section{Mobile clinics}

Lilleston et al. (2018) delivered a mobile SGBV service targeting Syrian refugee women in Lebanon [31]. Three mobile teams each consisted of a community mobiliser, a caseworker, and an adolescent girls' assistant. All team members were women, except for one male community mobiliser who rotated between the three teams [31]. Each mobile team also included an adolescent girls' assistant, whom the girls said provided them with emotional support. This service aimed to complement existing static services and provided activities that addressed psychosocial issues and risk mitigation as well as individual case management.

\section{Integration with non-SRH services}

In Rwanda, the Uyisenga N'Manzi intervention aimed to reduce HIV risk amongst its participants by integrating HIV prevention education into an existing mental health intervention. This intervention included group discussions, lectures and presentations from staff covering sexuality, HIV and prevention strategies [30].

\section{Additional strategies to increase utilisation of SRH services}

In Colombia, Bosmans et al (2012) used the arts as a form of health education [29]. Interactive theatre, dancing, painting and singing were used to promote body awareness and address SRH issues, including taboo topics, among internally displaced youth [29].

In Sierra Leone, Barnett et al. (2018) adapted preexisting radio programme to continue to educate youth when schools shut down during a crisis [34]. This included a radio programme aimed at $12-18$ year olds that aimed to increase awareness of prevention of teen pregnancy, HIV and violence [34].

\section{Implementers and donors of SRH interventions}

Over half $(n=9)$ of the $15 \mathrm{SRH}$ interventions targeted to young people in this review were implemented by large international agencies e.g. UNFPA, IRC, WRC, ARC International, WHO and Save the Children, usually in collaboration with local authorities and organisations. Information on donors for SRH interventions in humanitarian settings was only reported for 8 of the 15 interventions, and included the David and Lucille Packard Foundation [27, 40], the Department for International Development (DFID) [28, 39], the National Institute of Mental Health [32, 33], Gilead [30], and the Government of Belgium [29]. One intervention was funded as a collaboration between the US Department of State, NoVo
Foundation and the Swedish International Development Cooperation [31].

\section{Key findings}

Key findings from each study are presented in Table 3. The majority of studies measured output data (e.g. number of services provided, utilisation rates), and changes in knowledge and attitudes, whereas fewer studies measured changes in behaviour or risk. Nearly all studies $(n=13)$ reported some positive SRH outcomes, the majority of which were positive changes in knowledge and attitudes, half reported no effects in some SRH outcomes measured $(n=7)$, and one study reported a decrease in number of new and repeat contraceptive clients.

\section{Discussion}

This is the first systematic review to assess the evidence base on SRH interventions with health outcome data for young people, including adolescents, in humanitarian settings. All but one of the 14 included articles were published in the previous 7 years, showing that studies on SRH interventions for young people in humanitarian settings have only recently started to become a priority, which is consistent with global trends related to research and programming on health services for young people [9].

The review included a range of study designs with mostly high to medium quality, which is consistent with previous reviews on SRH interventions in humanitarian settings $[3,17]$. While the quality of the articles could be assessed through the use of critical appraisal tools, we were unable to determine the quality of individual SRH interventions. This is because, while programme reporting standards exist [41], these were not adhered to in the included articles.

There are known challenges to conducting research in humanitarian settings. These challenges include security risks for researchers, lack of resources to conduct research and instability and mobility of the study population [3]. However, a previous review of health care services in humanitarian settings found high-quality studies in the fields of mental health and communicable diseases which suggests that it is possible for good research to be done in these settings [19]. Another barrier to conducting research may be the special ethical considerations that need to be made when working with young people and SRH, as described by WHO [42]. Legal requirements may require parental consent for participation in research, especially for younger adolescents, which may be a challenge for recruitment in humanitarian settings, where young people are often separated from their parents.

This systematic review found few studies of SRH interventions for young people in humanitarian settings, similar to that found in previous reviews of SRH 
interventions in humanitarian settings [3, 17]. Our findings show that while there has been an increase in conducting research and documenting programming in this field in recent years, we need better quantity and quality of data using rigorous evaluation methods on SRH interventions for young people and adolescents. These data should be collected and analysed via standardised monitoring systems, with integration between humanitarian and local health information systems where possible and appropriate. It is important that when SRH data are collected, they are disaggregated by age using standard age groups in order to understand gaps in SRH service provision for this population in humanitarian settings and to make evidence-based decisions when allocating financial resources.

Over half $(n=9)$ of the interventions included in this review were described in the grey literature and all of these were from reports produced by large international non-governmental organisations (NGOs). The interventions included may therefore not be a good representation of all SRH interventions for young people in humanitarian settings but rather reflect the main funders and donors active in this field. It may be the case that those interventions implemented by local governments and smaller organisations are less likely to be documented, evaluated and published due to capacity and resource constraints. NGOs may not always prioritise the publication of programme results, especially in peerreviewed literature. The lack of studies in this field could therefore be due to challenges related to the setting and population, which have resulted in rigorous programme evaluation not being the norm in these settings, or due to a lack of funding for this type of research.

The interventions included in our review were implemented in acute disease outbreak $(n=1)$, natural disaster $(n=3)$, protracted armed conflict $(n=8)$ and postconflict $(\mathrm{n}=3)$ and settings in LMIC. Interventions focused on prevention of unintended pregnancies, HIV and STIs, maternal and newborn health, and prevention of SGBV, with an emphasis on reproductive 'problems' i.e. pregnancy and HIV, and little focus on sexual health including comprehensive sexuality education. We found no studies focusing on PMTCT, urogenital fistulae or FGM. Consistent with previous reviews assessing SRH in humanitarian settings, we found no studies documenting the provision of safe abortion services and few studies documenting post-abortion care services being delivered $[3,17]$. None of the studies reported inclusion of young people from sexual minorities or those with disabilities. Strategies to increase utilisation of SRH interventions by young people include adolescent-friendly spaces, peer workers, school-based activities, and involving young people in the development, implementation and evaluation of interventions. Most of the positive SRH outcomes reported in the review showed an improvement in knowledge and attitudes, although these effects did not seem to always translate to changes in behaviour or risk status. This may be due to the short duration of implementation of the interventions, in most cases less than 12 months, which is likely insufficient to observe significant behavioural change. We found no data on economic evaluation of SRH interventions for young people in humanitarian crises settings.

The MISP is only intended to be implemented at the onset of an acute crises, and is meant to set the stage for a transition to comprehensive SRH services as the crisis stabilises [43]. These findings show that the limited spectrum of SRH services delivered in humanitarian settings has not changed since previous evaluations $[16,17]$. Even though the MISP has been criticised by some actors as being too comprehensive to deliver at the start of a crisis [44], we would argue that the interventions described in this package should at the very least be provided to populations including young people in stabilised post-conflict settings.

None of the included interventions targeted boys only and, of the interventions aimed at reducing SGBV, all targeted only girls. However, research has shown that interventions that attempt to have a more gender transformative approach including targeting both men and women are more effective, especially when it comes to influencing long-term changes in societal norms and changing gender power relations [45]. In addition, very few interventions were explicitly inclusive of very young adolescents, indicating that this group, whose needs vary from older adolescents, are not being prioritised.

Of the interventions aiming to decrease the risk of SGBV, only the COMPASS intervention provided any outcome data, which showed no change in exposure to SGBV. This may be because end line data was collected directly following the 10-month intervention which may not have been a long enough time period to see real societal change resulting in decreased SGBV. Therefore, consideration needs to be given on how to assess the effectiveness of interventions like these targeting community-level change in an unstable and rapidly changing population.

Several interventions reported initial community resistance to the provision of SRH services to young people. Adults have been shown to influence the healthseeking behaviour of young people and, as such, it is essential that any new interventions have the support of the adult community and are seen as beneficial to this population [46]. It is also encouraging that many of interventions in this review reported involvement of young people at some point in the intervention cycle. Evidence has shown that meaningful engagement with and participation from young people increases positive outcomes of this population using health services [47]. 
There are a number of limitations to this systematic review. Firstly, due to the lack of published information on this topic, it is difficult to draw definitive conclusions. While the inclusion of grey literature expanded the evidence available for the review, it may have biased the types of interventions included as larger humanitarian organisations are more likely have the resources to write and publish reports. Therefore, it is important to note that what has been published may not represent which SRH interventions are actually being implemented for young people in humanitarian settings. Additionally, some implementing agencies prioritise research and publications more than others, and seek funding and partnerships specifically for research, e.g. this review includes four peer-reviewed articles (describing three interventions) with collaborators from academic institutions.

While we were able to assess the spectrum of SRH interventions delivered to young people, as well as their effectiveness, utilisation, and implementation modalities to a certain extent, limited data in the included studies did not allow us to assess distal determinants including relevant national, institutional, political, legal, and cultural factors which likely influenced the uptake and impact of these SRH interventions.

Additionally, we may have excluded relevant studies due to only including English and French language articles based on the study team's capacity. We also excluded a number of articles, particularly in the grey literature, due to inadequate intervention descriptions and a lack of data on utilisation rate or health outcomes $[38,48,49]$.

\section{Conclusions}

This review highlights the need for a higher quantity and quality of studies documenting interventions addressing the comprehensive sexual and reproductive health needs of young people in their diversity in a range of humanitarian settings, including in acute and protracted conflict and natural disaster areas. While there is evidence that some SRH interventions for young people are being implemented, there are insufficient details of specific intervention components and outcome measurements to be able to adequately describe and assess these interventions. Implementers are using a number of different strategies including peer workers, adolescent-friendly settings and participatory approaches to increase utilisation of services by young people in humanitarian settings. However, there is a lack of research evaluating these interventions, which has implications for scale-up and sustainability. Interventions implemented in humanitarian settings in the future must invest in better documentation and evaluation.

\section{Supplementary information}

Supplementary information accompanies this paper at https://doi.org/10. 1186/s13031-019-0240-y.

Additional file 1. Search terms for systematic review of sexual and reproductive health interventions for young people including adolescents in humanitarian settings.

\begin{abstract}
Abbreviations
ASRH: Adolescent sexual and reproductive health; FGM: Female genital mutilation; IAFM: Inter-Agency Field Manual; IAWG: Inter-Agency Working Group; ICPD: International Conference on Population and Development; IRC: International Rescue Committee; LMIC: Low- and middle-income countries; MISP: Minimum Initial Services Package; PMTCT: Prevention of motherto-child transmission; PRISMA: Preferred Reporting Items for Systematic Reviews and Meta-Analyses; RCT: Randomised controlled trial; SGBV: Sexual and gender-based violence; SRH: Sexual and reproductive health; STI: Sexually transmitted infection; UNFPA: United Nations Population Fund; UNHCR: United Nations High Commissioner for Refugees; WHO: World Health Organization; WRC: Women's Refugee Commission
\end{abstract}

\section{Acknowledgments}

We would like to thank the IAWG adolescent SRH sub-working group for helping to identify relevant websites and resources to search for this review.

\section{Authors' contributions}

NSS and $L$ conceived the analysis and all authors contributed to the final structure and content of the paper. LJ and NSS led the overall data analysis, and $A S G, T J$ and $K B$ contributed to specific sections. $L J$ wrote the first draft of the paper with inputs from NSS, and all authors contributed to critical interpretation of the results and development of the paper. All authors saw and approved the final version.

\section{Funding}

We received funding from Countdown 2030 supported by the Bill and Melinda Gates Foundation (award number OPP1148933). Salary support for NSS is also provided under the RECAP project by the United Kingdom Research and Innovation as part of the Global Challenges Research Fund, grant number ES/P010873/1. LJ was supported by a UK Chevening Scholarship. ASG is supported by the South African Research Chair's Initiative of the Department of Science and Technology and National Research Foundation of South Africa (Grant No 82769) and the South African Medical Research Council. None of the funders had any role in study design, data collection, data analysis, data interpretation, or writing of the manuscript.

\section{Availability of data and materials}

All data generated or analysed during this study are included in this published article.

\section{Ethics approval and consent to participate}

The Research Governance and Integrity Office of the London School of Hygiene and Tropical Medicine assessed the study protocol and determined that an ethics approval was not required for a systematic review.

\section{Consent for publication}

Not applicable.

\section{Competing interests}

The authors declare that they have no competing interests.

\section{Author details}

${ }^{1}$ Health in Humanitarian Crises Centre, London School of Hygiene and Tropical Medicine, 15-17 Tavistock Place, London WC1H 9SH, UK. ${ }^{2}$ School of Public Health, University of the Western Cape, Cape Town, South Africa. 
Received: 18 July 2019 Accepted: 13 November 2019 Published online: 27 November 2019

\section{References}

1. United Nations Office for the Coordination of Humanitarian Affairs (OCHA) Global Humanitarian Overview 2018. 2018.

2. UNHCR. Global trends: forced displacement in 2018: UNHCR: Statistics On Refugees; 2018. Available from: https://www.unhcr.org/globaltrends2018/.

3. Warren E, Post N, Hossain M, Blanchet K, Roberts B. Systematic review of the evidence on the effectiveness of sexual and reproductive health interventions in humanitarian crises. BMJ Open. 2015:5(12):1-9.

4. Kerner B, Manohar S, Mazzacurati C, Tanabe M. Adolescent sexual and reproductive health in humanitarian settings. Forced Migr Rev. 2012; 40(August):21-2

5. UNFPA. Adolescent Sexual and Reproductive Health Toolkit for Humanitarian Settings. 2009.

6. Dick B, Ferguson BJ. Health for the world's adolescents: a second chance in the second decade. J Adolesc Health. 2015;56:3-6.

7. Bearinger LH, Sieving RE, Ferguson J, Sharma V. Global perspectives on the sexual and reproductive health of adolescents: patterns, prevention, and potential. Lancet. 2007;369:1220-31.

8. Viner RM, Ozer EM, Denny S, Marmot M, Resnick M, Fatusi A, et al. Adolescence and the social determinants of health. Lancet. 2012;379:1641-52.

9. Patton GC, Sawyer SM, Santelli JS, Ross DA, Afifi R, Allen NB, et al. Our future: a Lancet commission on adolescent health and wellbeing. Lancet. 2016:387:2423-78.

10. Schlecht J, Lee C, Kerner B, Greeley M, Robinson C. Prioritizing programming to address the needs and risks of very young adolescents: a summary of findings across three humanitarian settings. Confl Health. 2017;11(Suppl. 1):31.

11. Casey SE, Chynoweth SK, Cornier N, Gallagher MC, Wheeler EE. Progress and gaps in reproductive health services in three humanitarian settings: mixedmethods case studies. Confl Health. 2015;9(Suppl 1):S3.

12. United Nations. International Conference on Population and Development: Programme of Action. 2014 [cited 2018 Aug 22]. Available from: https://www.unfpa.org/publications/international-conferencepopulation-and-development-programme-action

13. Inter-Agency Working Group on Reproductive Health in Crises (IAWG) Inter-Agency Fleld Manual on Reproductive Health In Humanitarian Settings. 2018.

14. Foster AM, Evans DP, Garcia M, Knaster S, Krause S, McGinn T, et al. The 2018 inter-agency field manual on reproductive health in humanitarian settings: revising the global standards. Reprod Health Matters. 2017;25(51): $18-24$.

15. Inter-Agency Working Group on Reproductive Health in Crises. Interagency Field Manual on Reproductive Health in Humanitarian Settings. 2010.

16. Casey SE. Evaluations of reproductive health programs in humanitarian settings: a systematic review. Confl Health. 2015;9(1):S1

17. Singh NS, Aryasinghe S, Smith J, Khosla R, Say L, Blanchet K, et al. A long way to go: a systematic review to assess the utilisation of sexual and reproductive health services during humanitarian crises; 2018. p. 1-12.

18. Moher D, Liberati A, Tetzlaff J, Altman D. Preferred reporting items for systematic reviews and meta-analyses: the PRISMA statement. Ann Intern Med. 2009:151(4):264-9.

19. Blanchet K, Ramesh A, Frison S, Warren E, Hossain M, Smith J, et al. Evidence on public health interventions in humanitarian crises. Lancet. 2017:390: 2287-96.

20. Petticrew M, Rehfuess E, Noyes J, Higgins JPT, Mayhew A, Pantoja T, et al. Synthesizing evidence on complex interventions: how meta-analytical, qualitative, and mixed-method approaches can contribute. J Clin Epidemiol. 2013:66(11):1230-43.

21. Schulz KF, Altman DG, Moher D. CONSORT 2010 statement: updated quidelines for reporting parallel group randomised trials. BMC medicine. 2010;8(1):18.

22. von Elm E, Altman DG, Egger M, Pocock SJ, Gøtzsche PC, Vandenbroucke $J P$. The Stengthening the reporting of observational studies in epidemiology (STROBE) statement: guidelines for reporting observational studies. J Clin Epidemiol. 2008;61(4):344-9.
23. Study Quality Assessment Tools | National Heart, Lung, and Blood Institute (NHLBI) [Internet]. [cited 2018 Aug 23]. Available from: https://www.nhlbi. nih.gov/health-topics/study-quality-assessment-tools

24. Critical Appraisal Skills Programme (CASP) Checklist. 2018. https://casp-uk net/casp-tools-checklists/.

25. Center for Evidence-Based Medicine. Critical Appraisal of a Case Study [Internet]. [cited 2018 Aug 23]. Available from: https://www.cebma.org/wpcontent/uploads/Critical-Appraisal-Questions-for-a-Case-Study.pdf

26. Tyndall J. The AACODS checklist: Flinders University; 2010.

27. Casey SE, Larsen MM, McGinn T, Sartie M, Dauda M, Lahai P, et al. Changes in HIV/AIDS/STI knowledge, attitudes, and behaviours among the youth in Port Loko, Sierra Leone. Glob Public Health. 2006;1(3):249-63.

28. Stark L, Seff I, Assezenew A, Eoomkham J, Falb K, Ssewamala FM. Effects of a social empowerment intervention on economic vulnerability for adolescent refugee girls in Ethiopia. J Adolesc Health. 2018;62:S15-20.

29. Bosmans M, Gonzalez F, Brems E, Temmerman M. Dignity and the right of internally displaced adolescents in Colombia to sexual and reproductive health. Disasters. 2012;36(4):617-34.

30. Talbot A, Uwihoreye C, Kamen C, Grant P, McGlynn L, Mugabe I, et al. Treating psychological trauma among Rwandan orphans is associated with a reduction in HIV risk-taking behaviors: a pilot study. AIDS Educ Prev. 2013;25(6):468-79.

31. Lilleston P, Winograd L, Ahmed S, Salame D, Al Alam D, Stoebenau K, et al. Evaluation of a mobile approach to gender-based violence service delivery among Syrian refugees in Lebanon. Health Policy Plan. 2018;33:767-76.

32. Atwood KA, Kennedy SB, Shamblen S, Taylor CH, Quaqua M, Bee EM, et al. Reducing sexual risk taking behaviors among adolescents who engage in transactional sex in post-conflict Liberia. Vulnerable Child Youth Stud. 2012; 7(1):55-65.

33. Atwood KA, Kennedy SB, Shamblen S, Tegli J, Garber S, Fahnbulleh PW, et al. Impact of school-based HIV prevention program in post-conflict Liberia. AIDS Educ Prev. 2012;24(1):68-77.

34. Barnett S, van Dijk J, Swaray A, Amara T, Young P. Making Multisectoral Collaboration Work: Redesigning an education project for child friendly radio: a multisectoral collaboration to promote children's health, education, and human rights after a humanitarian crisis in Sierra Leone. The BMJ. 2018; 363: k4667. https://doi.org/10.1136/bmj.k4667.

35. Chaudhary P, Vallese G, Thapa M, Alvarez V, Pradhan L, Bajracharya K, et al. Humanitarian response to reproductive and sexual health needs in a disaster: the Nepal earthquake 2015 case study. Reprod Health Matters. 2017;25(51):25-39.

36. UNFPA. Adolescent Girls in Disaster \& Conflict: Interventions for Improving Access to Sexual and Reproductive Health Services. 2016.

37. Tanabe M, Modigell I, Manohar S. CASE STUDY : adolescent sexual and reproductive health programming in Goma, Democratic Republic of the Congo; 2013

38. Tanabe M, Schlecht J, Manohar S. Adolescent sexual and reproductive health programs in humanitarian settings: an in-depth look at family planning services. New York; 2012. https://www.unfpa.org/sites/default/files/ resource-pdf/AAASRH_good_practice_documentation_English_FINAL.pdf.

39. Tanner S, O'Conner M. A safe place to Shine: creating opportunities and Rasing voices of adolescent girls in humanitarian settings; 2017.

40. Nehme R, Spilotros N. She knows best: engaging girls in adolescent programming. New York; 2018. https://www.rescue.org/report/she-knowsbest-engaging-girls-adolescent-programming.

41. Kågesten AE, Tunçalp Ö, Portela A, Ali M, Tran N, Gülmezoglu AM. Programme Reporting Standards (PRS) for improving the reporting of sexual, reproductive, maternal, newborn, child and adolescent health programmes. BMC Med Res Methodol. 2017;17(1):117.

42. World Health Organization. Guidance on ethical considerations in planning and reviewing research studies on sexual and reproductive health in adolescents. 2018

43. Krause SK, Chynoweth SK, Tanabe M. Sea-change in reproductive health in emergencies: how systemic improvements to address the MISP were achieved. Reprod Health Matters. 2017:25(51):7-17.

44. Tran NT, Schulte-Hillen C. Wishful thinking versus operational commitment: is the international guidance on priority sexual and reproductive health interventions in humanitarian settings becoming unrealistic? Confl Health. 2018:12:32.

45. Fulu E, Kerr-Wilson A, Lang J, Gibbs A, Jacobson J, Jewkes R, et al. What works to prevent violence against women and girls? Evidence review of interventions to prevent violence against women and girls. 2014. 
46. Inter-Agency Working Group (IAWG) on the Role of Community

Involvement in ASRH. Community Pathways to Improved Adolescent Sexual and Reproductive Health: A Conceptual Framework and Suggested

Outcome Indicators. Washington and New York; 2007. https://www.unfpa. org/sites/default/files/resource-pdf/asrh_pathways.pdf.

47. Melles MO, Ricker CL. Youth participation in HIV and sexual and reproductive health decision-making, policies, programmes: perspectives from the field. Int J Adolesc Youth. 2018;23(2):159-67.

48. Caton C, Chaffin J, Marsh M, Read-Hamilton S. Empowered and safe economic strengthening for girls in emergencies; 2014

49. Paik K. Strong Girls, Powerful Women: Program Planning and Design for Adolescent Girls in Humanitarian Settings. New York; 2014. https:/www. womensrefugeecommission.org/images/zdocs/Strong-Girls--PowerfulWomen--2014.pdf.

\section{Publisher's Note}

Springer Nature remains neutral with regard to jurisdictional claims in published maps and institutional affiliations.

Ready to submit your research? Choose BMC and benefit from:

- fast, convenient online submission

- thorough peer review by experienced researchers in your field

- rapid publication on acceptance

- support for research data, including large and complex data types

- gold Open Access which fosters wider collaboration and increased citations

- maximum visibility for your research: over $100 \mathrm{M}$ website views per year

At BMC, research is always in progress.

Learn more biomedcentral.com/submissions 